\title{
ResearchOnline@JCU
}

This is the author-created version of the following work:

Thorley, Craig (2018) Enhancing individual and collaborative eyewitness memory with category clustering recall. Memory, 26 (8) pp. 1128-1139.

\author{
Access to this file is available from: \\ https://researchonline.jcu.edu.au/52119/
}

Please refer to the original source for the final version of this work:

https://doi.org/10.1080/09658211.2018.1432058 


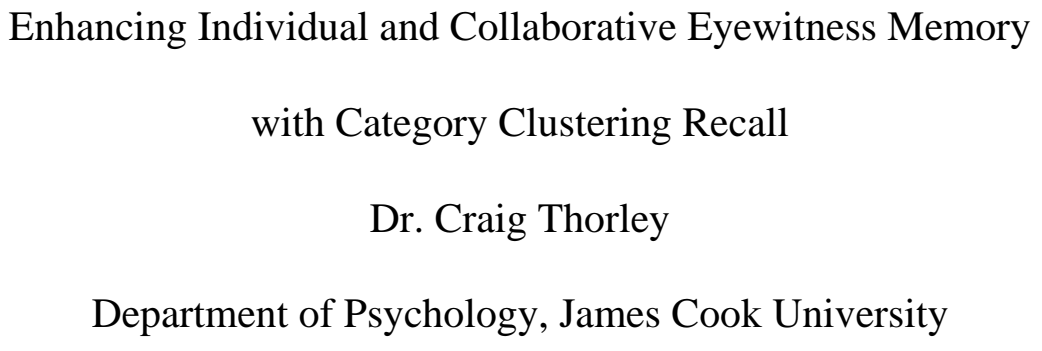

Address for correspondence:

Dr. Craig Thorley

Department of Psychology

James Cook University

Townsville, 4814

Australia

Phone: +61 747816354

Email: craig.thorley@jcu.edu.au

Acknowledgements: Thanks to Aaron Ewart, Danielle Goodwin, Grace Hunt, Anniken Slyngenborg Jensen, Devvarta Kumar, and Douglas McCombie for assistance with data collection and scoring. Thanks also to Kate A. Houston for providing the crime video used in this study. 


\begin{abstract}
Most crimes have multiple eyewitnesses. The police typically interview co-witnesses separately. In time sensitive investigations, this could slow down evidence accumulation. Having co-witnesses collaboratively recall a crime could potentially expedite evidence accumulation. However, past research shows collaborative group members often have conflicting retrieval strategies that disrupt each other, degrading overall recall. This cost could potentially be overcome by aligning group members' retrieval strategies with Category Clustering Recall (CCR), which is a retrieval strategy where information is recalled from a series of forensically relevant categories (e.g., recalling the protagonists' appearance, then actions). This study examined the costs and benefits of collaborative eyewitness memory by having collaborative pairs of strangers, nominal pairs (i.e., two individuals whose recall is pooled) and lone individuals watch a crime and recall it using free recall or CCR. The collaborative pairs recalled the crime faster than the nominal pairs. They also recalled more correct information than individuals but less than nominal pairs, irrespective of the retrieval method. There is therefore a speed-recall completeness trade-off when collaborative groups recall crimes. Importantly, all participants recalled more correct information when using CCR. This provides initial evidence suggesting CCR is superior to free recall. Further research examining CCR's benefits is recommended.
\end{abstract}

Keywords: collaborative memory, eyewitness memory, category clustering recall, collaborative inhibition, retrieval strategy disruption 


\section{Enhancing Individual and Collaborative Eyewitness Memory with Category Clustering Recall}

Most crimes have multiple eyewitnesses (Paterson \& Kemp, 2006; Skagerberg \& Wright, 2008). It is often recommended that the police separate co-witnesses and interview them alone (e.g., National Institute of Justice, 1999). Doing so ensures they cannot contaminate each other's memories of the crime (Wright, Memon, Skagerberg, \& Gabbert, 2009). If, however, a large number of individual statements need to be taken, this could slow down the rate at which evidence is accumulated and an offender is apprehended. This would be problematic if there was a strong possibility of a further offence being committed. In these circumstances, evidence may be accumulated faster if co-witnesses collaboratively recalled the crime. To date, few studies have investigated the costs and benefits of having cowitnesses collaboratively recall a crime shortly after witnessing it. The present study therefore examines these costs and benefits and also considers whether the completeness and accuracy of collaborative eyewitness recall can be maximised using a novel form of retrieval known as Category Clustering Recall (or CCR; see Paulo, Albuquerque, \& Bull, 2016; Paulo, Albuquerque, Vitorino \& Bull, 2017).

\section{Collaborative Eyewitness Memory (Correct Recall)}

Perhaps unsurprisingly, collaborative groups of eyewitnesses can recall more correct information about crimes than lone eyewitnesses (Wessel, Zandstra, Hengeveld, \& Moulds, 2015). This does not, however, mean that collaboration benefits recall. To truly understand whether collaboration enhances the volume of correct information eyewitnesses recall, collaborative groups must be compared to equivalent sized nominal groups. Nominal groups are created by the recall of several individuals and counting overlapping information once. They provide a measure of a collaborative group's potential output if collaboration neither inhibits nor facilitates recall. To date, two studies have found that collaborative groups recall 
less correct information about crimes than nominal groups (Bärthel, Wessel, Huntjens, \& Verwoerd, 2017; Wessel et al., 2015). This decrement is known as collaborative inhibition (or CI; Weldon \& Bellinger, 1997). Two other studies have, however, found they recall as much correct information about crimes as nominal groups (Vredeveldt, Hildebrandt, \& Van Koppen, 2016; Vredeveldt, Groen, Ampt \& van Koppen, 2017).

To understand why some collaborative eyewitness memory studies have observed CI and others have not, a brief overview of the wider collaborative memory literature is needed. In that wider literature, dozens of studies have compared collaborative and nominal groups' recall of simple materials such as word lists and stories (see Rajaram, 2011, for a review). Consistent with the eyewitness memory studies just discussed, they have found that collaborative groups recall more correct information than individuals (see Clark \& Stephenson, 1989, for a review) and that they sometimes, but not always, recall less correct information than nominal groups.

Whether CI occurs depends, at least in part, on the degree of retrieval strategy disruption (RSD) present during collaborative remembering (Basden, Basden, Bryner, \& Thomas, 1997; see Marion \& Thorley, 2016, for a review and meta-analysis) ${ }^{1}$. According to RSD theory, collaborative group members can have idiosyncratic, but optimal, retrieval strategies when recalling studied information. For example, when recalling a crime, Witness A may prefer to recall an offender's actions first, whereas Witness B may prefer to describe an offender's appearance first. When collaborative group members attempt to recall information using different retrieval strategies, these conflicting strategies disrupt each other

\footnotetext{
${ }^{1}$ It is generally acknowledged in the collaborative memory literature that retrieval strategy disruption is a leading cause of collaborative inhibition but that other cognitive mechanisms also contribute to the effect (e.g., see Hyman, Cardwell \& Roy, 2013; Barber, Harris, \& Rajaram, 2015).
} 
and the productivity of the group suffers. Consequently, the collaborative group members each recall less than they would if working alone, resulting in CI.

The degree of RSD experienced by a collaborative group is mediated by a number of factors including its size and the social relationship of its members (Marion \& Thorley, 2016). Importantly, those collaborative eyewitness memory studies that observed CI (Bärthel et al., 2017; Wessel et al., 2015) and those that did not (Vredeveldt et al., 2016; 2017) differed with respect to both factors. Group size matters, as larger groups are more prone to CI (e.g., Marion \& Thorley, 2016; Thorley \& Dewhurst, 2007). This occurs as larger collaborative groups have a greater number of conflicting retrieval strategies being used, meaning the RSD is more pronounced and the productivity of the group decreases (Basden, Basden, \& Henry, 2000). Social relationships matter as CI is greater in collaborative groups of strangers, relative to collaborative groups of close acquaintances (e.g., Andersson \& Rönnberg, 1995, 1996; Marion \& Thorley, 2016). One explanation for this is that some close acquaintances have a transactive memory system (Rajaram \& Pereira-Pasarin, 2010). This is a shared memory system for encoding, storing, and retrieving information that allows group members to utilise complementary, as opposed to conflicting, retrieval strategies, thus reducing CI. Several studies have also found that some collaborative groups of close acquaintances (e.g., married couples) have supportive communication styles that facilitate their recall and make them less prone to CI (e.g., Harris, Keil, Sutton, Barnier, \& McIlwain, 2011; Vredeveldt et al., 2016). Importantly, those past collaborative eyewitness memory studies that observed CI tested collaborative pairs of strangers (Bärthel et al., 2017; Wessel et al., 2015), whereas those that did not observe CI tested collaborative trios where all members (Vredeveldt et al., 2016) or most members (Vredeveldt et al., 2017) were close acquaintances. Thus, the larger group sizes/lack of social relationship between group 
members in Bärthel et al. (2017) and Wessel et al.’s (2015) studies meant their collaborative groups likely experienced greater RSD, resulting in CI.

A third, non-RSD related, difference between those collaborative eyewitness memory studies that observed CI and those that did not, that may have contributed to their conflicting findings, was their study-test delays. The two studies that found evidence of CI had a 5-min study-test delay only (Bärthel et al., 2017; Wessel et al., 2015) whereas those that found no evidence of CI had study-test delays exceeding 15 min (Vredeveldt et al., 2016, 2017). This is important as it has been demonstrated that $\mathrm{CI}$ is present during experiments that have study-test delays of up to 15 min (Takahashi \& Saito, 2004) but disappears in experiments with longer study-test delays of 2 hrs (Congleton \& Rajaram, 2011), 24 hours (Abel \& Bäuml, 2017), and 1 week (Takahashi \& Saito, 2004). Congleton and Rajaram (2011) and Abel and Bäuml (2017) both offer evidence to suggest CI decreases over time because of a nominal group disadvantage. More specifically, they found evidence that participants forget studied information over time but that collaborative groups are less susceptible to this forgetting as their members can provide each other with cues (in the form of retrieved items) that facilitate each other's recall. As nominal group members cannot communicate, they are unable to provide each other with these beneficial cues. The extent of cross-cuing evident in Abel and Bäuml’s (2017) study was small, raising the possibility that other factors may contribute towards the elimination of CI. Regardless, the longer study-test delays in Vredeveldt et al.’s (2016, 2017) studies likely gave their collaborative groups a recall advantage over those in Bärthel et al. (2017) and Wessel et al.'s (2015) studies, meaning their collaborative groups were less susceptible to CI.

\section{Reducing Collaborative Inhibition}

Taken together, the above research suggests the police should consider whether it is worthwhile asking stranger co-witnesses to collaboratively recall a crime in its immediate 
aftermath, given that the eyewitnesses would likely recall less correct information about it than they would individually. Collaboration would, however, likely expedite evidence accumulation in time-sensitive cases with large numbers of eyewitnesses. It would therefore be beneficial if stranger co-witnesses could collaboratively recall a crime in a way that overcame the problem of CI. One potential way of achieving this, which has been successful in the wider collaborative memory literature, would be to align group members retrieval strategies during collaboration.

In the wider collaborative memory literature, collaborative group members retrieval strategies have been aligned in several different ways. In the first study to do this, Basden et al. (1997, Experiment 4) had collaborative and nominal groups of strangers study categorised word lists. The groups were then asked to either freely recall the items in any order they wished or to recall all items from each category, one category at a time. When the collaborative groups could freely recall the items in any order they wished, their members utilised different retrieval strategies and CI occurred. When the collaborative groups recalled items from one category at a time, their members' retrieval strategies were more aligned and CI was eliminated. In a second study, Saraiva, Albuquerque and Arantes (2016) had collaborative and nominal groups of strangers study unrelated words. They were then asked to freely recall the words in any order they wished or to recall the words using serial recall (meaning group members were all recalling items using the same retrieval strategy). CI was evident during free recall but not serial recall. In a third study, Finlay, Hitch, and Meudell (2000) had collaborative and nominal groups of strangers study word-pairs. They were then asked to freely recall the words in any order or were given a cued recall test in which one word-pair member was presented and they had to remember the non-presented member. Thus, the latter condition aligned group members retrieval strategies by forcing them to recall items in the same order. CI was evident during free recall but not cued recall. 
As aligning collaborative group members retrieval strategies when they are recalling word lists can eliminate CI, it is possible that aligning them when they recall a crime would have a similar effect. This alignment could be achieved by having collaborative groups of eyewitnesses engage in Category Clustering Recall (CCR), which is comparable to the category-by-category recall used by Basden et al. (1997, Experiment 4).

\section{Category Clustering Recall}

CCR was recently introduced into the eyewitness memory literature by Paulo and colleagues (Paulo et al., 2016, 2017). This new retrieval strategy involves presenting lone eyewitnesses with several crime-relevant category labels, one at a time, and asking them to recall as much information as possible from these categories. For example, eyewitnesses may first be asked to recall the physical descriptions of the people involved in the crime, then their actions, then the crime environment. CCR was not intended to be a standalone retrieval strategy. Paulo et al.’s studies were primarily interested in examining whether eyewitnesses who recall a crime during a Cognitive Interview would remember more about it if that interview was amended so that it also contained CCR. For readers unfamiliar with the Cognitive Interview, it is a police interview technique developed by Fisher and Geiselman (1992) that contains several recall enhancing mnemonics (see Geiselman \& Fisher, 2014, or Paulo, Albuquerque, \& Bull, 2013, for overviews). A key feature here is that during the Cognitive Interview, eyewitnesses are initially asked to freely recall a crime and this is then followed by a second recall attempt that can involve changing the order of recall (e.g., recalling the crime in reverse chronological order) or witness compatible questioning (e.g., asking the witness open-ended questions compatible with their previous recall and retrieval pattern). In their studies, Paulo et al. examined whether replacing these second interview options with CCR would produce a more complete account of the crime. It was found that using CCR during this second recall attempt produced additional information not retrieved 
during the initial free recall and the volume obtained was greater than with either the change order protocol (Paulo et al., 2016) or witness-specific questioning (Paulo et al., 2017). Moreover, CCR failed to increase the number of recall errors in either study. CCR is therefore an effective retrieval technique.

Paulo et al. (2016, 2017) speculate that CCR is effective as people naturally/spontaneously encode, organise, and retrieve information in semantic categories (Dalrymple-Alford \& Aamiry, 1969; Manning \& Kahana, 2012; Robinson, 1966). Asking eyewitnesses to recall information in categories that are aligned with their spontaneous subjective organisation may be less cognitively demanding for them, enhancing their recall. Moreover, asking them to successively recall information from one category cluster may induce spreading-activation that triggers memories of other details associated with that cluster that may otherwise not be retrieved, thus facilitating recall (Collins \& Loftus, 1975).

\section{Collaborative Eyewitness Memory (Recall Errors)}

So far, the literature review has focussed on collaboration's impact on correct recall but, from an applied perspective, it is also important to consider its impact upon recall errors. As mentioned, police guidelines often recommend separating multiple eyewitnesses and taking individual statements from them. On face value, this guidance is sensible as cases exist where real eyewitnesses have been exposed to co-witness misinformation about a crime and this misinformation has tainted their subsequent legal testimonies (see Wright et al., 2009; Gabbert, Wright, Memon, Skagerberg, \& Jamieson, 2011). When one person changes their account of an event to be consistent with another's differing account of the same event, they are said to have engaged in memory conformity (Wright, Self, \& Justice, 2000) or social contagion (Roediger, Meade, \& Bergman, 2001). The former term is favoured here. Memory conformity has been induced in the laboratory in dozens of studies by asking participants to engage in collaborative remembering with an intentionally erroneous confederate (e.g., 
Roediger et al., 2001) or a co-participant who witnessed a slightly different version of the same event (e.g., Gabbert, Memon, \& Allan, 2003). In each case, participants incorrectly claim to remember studying information that they did not personally study but that was recalled by their collaborative partner.

Whilst memory conformity can occur, it is important to emphasise that it has primarily been observed in studies where a researcher intentionally induces it. In the collaborative memory literature, researchers do not typically try to induce recollection errors (see Thorley \& Dewhurst, 2007, 2009, for exceptions) and it has consistently been found that collaborative groups make few recall errors overall. This likely occurs as free recall requires self-guided retrieval, meaning people typically only report information they are confident about (Koriat \& Goldsmith, 1994, 1996). Moreover, when errors are found, collaborative groups tend to make fewer than nominal groups. This occurs irrespective of whether participants are recalling simple materials, such as word lists, or more complex events, such as a crime (e.g., Harris, Barnier, \& Sutton, 2012, 2013; Ross, Spencer, Blatz, \& Restorick, 2008; Vredeveldt et al., 2016, 2017; Weigold, Russell, \& Natera, 2014; Wessel et al., 2015). One reason why collaborative groups make fewer recall errors is that their members correct each other’s mistakes during collaboration (Ross et al., 2008). Thus, having co-witnesses recollect a crime together may reduce the number of errors made.

\section{The Present Study}

In this study, individuals, collaborative pairs of strangers, and nominal pairs will watch a crime video and then, after a short delay, recollect the crime using either free recall or CCR. Their recall completeness, which here refers to the number of correct details they can recall, and their recall accuracy, which here refers to the number of recall errors they make, will be assessed. 
This design allows several novel research questions to be answered. First, it will help determine whether having collaborative pairs recall a crime shortly after it has occurred can speed up the rate at which evidence is accumulated (in comparison to when two eyewitnesses recall the crime separately). This is forensically important to know as situations can arise where statements need to be taken from large numbers of eyewitnesses and evidence needs to be accumulated as quickly as possible to try and prevent a further offence being committed. Second, it will indicate whether recalling alone results in less complete and accurate recall, relative to recalling in a collaborative pair, irrespective of the retrieval method used. Third, and more importantly, it will help determine whether collaborative pairs who engage in CCR, relative to those who engage in free recall, are less prone to CI. This is forensically important as any results showing that engaging in CCR makes collaborative pairs immune to CI would provide initial evidence that it would be worthwhile taking statements from pairs of eyewitnesses using this retrieval technique in future. Fourth, it will help determine whether individuals, collaborative groups, and nominal groups have more complete and accurate recall when engaging in CCR or free recall. To date, CCR has not been directly compared to free recall. Of these comparisons, it is helpful to know whether engaging in CCR enhances lone eyewitness's recall. If so, this would provide initial evidence suggesting it is more profitable to interview lone eyewitnesses with this retrieval technique.

It is possible to form several hypotheses for this study. Common sense dictates that it will take longer for two separate eyewitnesses (whose data will be pooled to form a nominal group) to recall a crime than a collaborative pair as the nominal group members will likely recall some of the same information twice whereas the collaborative group members will only recall it once. Consistent with the discussed past research, it is also expected that individuals will freely recall fewer correct crime details than the collaborative and nominal pairs. Also in line with the discussed past research, the collaborative pairs are expected to 
freely recall fewer correct crime details than the nominal pairs, signifying CI. Any hypotheses involving CCR are tentative given that it has only previously been used in studies with individuals and when it followed free recall. Given that individuals typically remember fewer correct details than collaborative and nominal pairs during free recall, it is likely this will also occur during CCR. If CCR does align collaborative group members retrieval strategies, then it is possible that collaborative pairs using this method will recall as many correct details as nominal pairs, signifying no CI. Given that past research by Paulo et al. (2016, 2017) also found that engaging in CCR after free recall resulted in additional correct crime details being remembered, it is possible that it is a superior recall method. It is therefore tentatively predicted that individuals, collaborative pairs, and nominal pairs who use CCR will have more complete recall of the crime than their counterparts who use free recall. Finally, it is anticipated that collaborative pairs will have more accurate recall than either individuals or nominal pairs, irrespective of the recall method used, due to error correction. There are no grounds to expect any difference in the number of recall errors in the CCR conditions and free recall conditions.

\section{Method}

\section{Participants and Design}

200 participants (167 females, 33 males), aged $18-55(M=22.34, S D=7.12)$, were recruited via online and poster advertisements distributed within a university. They were compensated with course credit or a small honorarium.

The study had a between-subjects design with six conditions that differed according to Group Type (individuals; collaborative pairs; nominal pairs) and Recall Type (free recall; CCR). There were 20 participants in the individual free recall condition, 20 in the individual CCR condition, 20 pairs of participants in the collaborative free recall condition and 20 pairs of participants in the collaborative CCR condition. Members of two collaborative pairs were 
previously acquainted as they were studying the same university degree but had only known each other for four months.

A further 80 participants worked alone, with half engaging in free recall and the remainder in CCR. These participants were used to create the nominal groups. The data from the former 40 participants was randomly coupled, creating 20 pairs of participants in the nominal group free recall condition. The data from the latter 40 participants was also randomly coupled, creating 20 pairs of participants in the nominal group CCR condition. When creating these nominal groups, the same participant could only appear in one pair.

\section{Stimuli}

Participants watched a 2 min 32 s crime video taken from Houston, Clifford, Memon, and Phillips (2013). The video begins with an elderly woman withdrawing money from a cash machine and placing it in her handbag. She then walks down a residential street and enters a secluded wooded area with a pathway. Whilst walking through the wooded area, a man approaches her. The man grasps the woman with both hands and demands that she passes him her handbag. As the woman protests, the man forcefully removes the handbag off her shoulder and runs off with it. The woman then removes a mobile phone from her pocket and calls a friend to say she has been mugged.

Participants also watched a 10 min filler video, which was an excerpt from a nature documentary about a desert. Its content does not overlap with the crime video in any way.

\section{Procedure}

Participants were recruited individually and in pairs, with the advertisements telling them that the study was comparing individual and group decision making. Participants who were recruited individually were randomly allocated to one of the two Recall Type conditions. When participants were recruited in pairs and both turned up for the session, they were randomly allocated to either the collaborative free recall or collaborative CCR 
condition. If only one of the pair turned up for a session, that person was randomly allocated to one of the two Recall Type conditions.

All participants were initially told they would be required to watch two videos. No mention was made of any memory test. They were then sat at a computer (or separate computers, if tested in pairs), donned headphones, and watched the crime video. Immediately afterwards, they watched the filler video. All participants then received the memory test instructions. All were initially told that their memory of the first video, depicting the crime, was to be assessed and that they would be required to verbally recall it. They were also told that their recall would be digitally recorded. The instructions for participants in the free recall and CCR conditions then differed.

Lone participants in the individual/nominal pair free recall conditions were asked to freely recall as much information as possible from the video in any order and at any pace they wished (similar to the initial free report instructions in Paulo et al., 2016, 2017). Participants in the collaborative free recall conditions were asked to sit next to each other and received the same instructions but were asked to work together to freely recall as much information from the video as possible in any order and at any pace they desired. No guidance was given with regards to how they should collaborate (e.g., who should speak first) but they were asked to resolve any disagreements they may have by coming to a consensus about what they saw.

Lone participants in the individual/nominal pair CCR conditions were first asked to recall as much information as possible about the appearance of two main protagonists in the video, then their actions, and then the background environment in which the crime took place (henceforth called People Details, Action Details, and Environment Details, respectively). It was also emphasised they should do this at their own pace. Participants in the collaborative CCR conditions were asked to sit next to each other and received the same instructions but were asked to work together to recall information from each category. Again, no guidance 
was given with regards to how they should collaborate but they were asked to try and resolve any disagreements by coming to a consensus about what they saw. Once the participants had recalled as much information as possible, using either recall technique, they were debriefed and the study ended.

It is important to acknowledge that the CCR used here is analogous to, but not identical to, that used by Paulo et al. $(2016,2017)$ as they used several additional categories (specifically, their participants were asked about person details, person location details, object details, object location details, action details, conversation details, and sound details). The reason for this difference is that the present study commenced prior to the publication of Paulo et al.'s first, meaning the two approaches were developed independently and without any knowledge of each other.

\section{Data Scoring}

Participant’s digitally recorded recall was first scored for the amount of time, in secs, it took them to produce their statement, from the moment they first started recalling to the moment they finished recalling. Their recall was then scored for the number of correct and incorrect crime details remembered. To assist with this process, a coding scheme was created that utilised the three CCR categories already described (People Details, Action Details, Environment Details). The process involved in creating the coding scheme will be briefly described. First, three student assistants independently watched the crime video and created three independent coding schemes that listed as much information as possible about the physical descriptions of the two people involved in the crime (e.g., what colour their hair was; what colour clothing they wore), their actions (e.g., what the victim did prior to being mugged; what the offender did during the mugging), and the crime environment (e.g., where the crime took place; what objects appeared in the background). Second, these three coding schemes were pooled to create a single master coding scheme (with overlapping information 
included once). This master coding scheme contained 48 distinct People Details, 96 distinct Action Details, and 199 distinct Environment Details.

Each digital recording of the crime was scored against the master coding scheme by a student assistant who was blind to the aims of the study, with one point awarded for each correct People, Action, or Environment Detail recalled. For example, if a participant correctly stated "The man in a blue gilet was first seen walking near a fence in the wooded area. He then stopped in front of the woman and grabbed hold of her", then this was coded as "the man (People Detail) in a blue (People Detail) gilet (People Detail) was first seen walking (Action Detail) near a fence (Environment Detail) in the wooded area (Environment Detail). He then stopped in front of the woman (Action Detail for "stopped”; People Detail for “woman”) and grabbed hold of her (Action Detail)”. For the purposes of this analysis, the total number of People Details, Action Details, and Environment Details correctly recalled were scored (with the same information only scored once if it were repeated several times). These three scores were then tallied to provide an overall correct recall score. Recall errors were also scored. In this study, errors are details that appeared in the video but were described inaccurately (e.g., saying the man's gilet was red) or were not part of the video (e.g., a dog). Digressions, subjective remarks, or excessively vague details were not scored.

When scoring the collaborative groups' recall, only one group member needed to recall something correctly or incorrectly for it to be counted. If collaborative group members disagreed on a detail, their agreed response was recorded as the correct/incorrect answer. Similarly, in nominal groups, only one member needed to recall something correctly or incorrectly for it to be counted. Any overlapping information recalled by both nominal group members was scored only once. No instances arose where nominal group members contradicted each other (i.e., one member described a detail correctly but the other described it incorrectly). 
$25 \%$ of the eyewitness statements were double-scored by another student assistant. The inter-coder agreement between the first and second scorer was high $(\kappa=.89)$. The principal investigator resolved all disagreements by listening to the digital recording and ascertaining which coder was correct.

\section{Results}

\section{Time Taken to Recall the Crime}

One of the potential disadvantages of asking multiple eyewitnesses to produce separate statements, instead of collaborative group statements, is that doing so could slow down the rate at which evidence is accumulated. This initial analysis examined whether this disadvantage occurs by comparing how quickly collaborative and nominal groups recalled the crime. For nominal groups, the times taken by both members to recall the crime was summed. The time taken by individuals to recall the crime was also included in this analysis as a baseline measure. This analysis also examined whether the time taken by the three group types varied according to the recall type engaged in. On average, it took individuals $89.90 \mathrm{sec}$ $(S D=35.13)$ to freely recall the crime and $137.15 \mathrm{sec}(S D=38.33)$ to recall it using CCR. Collaborative pairs took longer than individuals, taking an average of $118.05 \sec (S D=$ 46.75) to freely recall the crime and $185.55 \mathrm{sec}(S D=49.65)$ to recall it using CCR. Most importantly, the nominal pairs took even longer, with an average of 211.10 sec $(S D=49.33)$ needed to freely recall the crime and $299.35 \mathrm{sec}(S D=55.59)$ needed to recall it using CCR.

A 3 x 2 between-subjects ANOVA, with Group Type and Recall Type as factors, would typically be employed to examine whether each condition significantly differed in the time taken to recall the crime. However, data screening, using the Shapiro-Wilks test for normality, revealed the time taken was non-normally distributed in several conditions due to skew (all $p$ 's<.05). Data transformations failed to correct the skew. As standard ANOVA performs poorly when data is non-normally distributed, the data was analysed with a robust 
version of the $3 \times 2$ ANOVA (Wilcox, 2016). The robust test used 20\% trimmed means and was performed in R (R Core Team, 2016) with the $t 2$ way function from Wilcox (2016) in the WRS2 package (Mair, Schoenbrodt, \& Wilcox, 2016). Post-hoc tests were carried out using the mcp2atm function from the same package. There was a main effect of Group Type, $Q=$ 130.05, $p<.001^{2}$, with individuals recalling the crime significantly quicker than both the collaborative pairs $(p=.002)$ and nominal pairs $(p<.001)$. Moreover, the collaborative pairs recalled the crime significantly quicker than the nominals pairs $(p<.001)$. There was also a main effect of Recall Type, $Q=48.16, p<.001$, with participants taking longer to recall the crime when using CCR than free recall $(p<.001)$. Finally, there was no Group Type x Recall Type interaction, $Q=2.39, p=.32$. To summarise the key findings, collaborative pairs can recall a crime quicker than two separate eyewitnesses and crimes are recalled quicker with free recall than CCR.

\section{Overall Correct Recall}

This analysis examined the impact of Group Type and Recall Type on overall correct recall. The Shapiro-Wilks test for normality showed the correct recall data in each of the six conditions was normally distributed (all p’s>.09) whereas Levene's test revealed the variances were homogenous $(p=.60)$. A 3 x 2 between-subjects ANOVA was therefore used to analyse this data. The Means and associated Standard Deviations can be seen in Table 1.

The ANOVA revealed a significant main effect of Group Type, $F(2,114)=22.02$, $p<.001, \eta p^{2}=.28$. Pairwise comparisons, with a Bonferroni adjustment lowering alpha to .017 , revealed fewer correct details were recalled by individuals $(M=20.32, S D=7.20)$ than collaborative pairs $(M=24.70, S D=7.93)(p=.01)$. They also revealed an overall effect of CI whereby collaborative pairs recalled fewer correct details than nominal pairs $(M=31.78$,

\footnotetext{
${ }^{2}$ Robust ANOVA tests in the WRS2 package produce a test statistic Q, which is approximately F-distributed. Degrees of freedom and effect sizes are not produced.
} 
$S D=8.95)(p<.001)$. As would be expected, given the previous two results, individuals also recalled fewer correct details than nominal pairs $(p<.001)$. The ANOVA also revealed a main effect of Recall Type, with participants recalling more correct details when engaging in CCR $(M=27.93, S D=8.37)$ than free recall $(M=23.27, S D=9.64)(F(1,114)=10.77, p=.001$, $\left.\eta_{p}^{2}=.09\right)$. Finally, there was no Group Type $\mathrm{x}$ Recall Type interaction, $F(2,114)=.03, p=$ $.76, \eta_{p}^{2}=.005$.

To summarise the most important findings, collaborative groups recalled more correct information than individuals, demonstrating two heads are better than one, but they also recalled less than nominal groups, demonstrating CI. Moreover, more correct details were recalled about the crime when participants engaged in CCR, irrespective of group type.

\section{INSERT TABLE 1 HERE}

\section{Correct Recall of People, Action, and Environment Details}

In exploratory analyses, the impact of Group Type and Recall Type on the correct recall of People, Action, and Environment Details was also examined. This was done to assess whether the effects observed in relation to overall correct recall extend to all three categories of forensically relevant information. The Means and associated Standard Deviations are in Table 1. Typically, this data would be analysed using a 3 x 2 MANOVA. Data screening, using the Shapiro-Wilks test for normality, revealed the data was nonnormally distributed in several conditions due to skew (all $p$ 's $<.05$ ). Data transformations failed to correct this issue, so it would be inappropriate to use MANOVA. Due to a lack of viable alternatives, each dependent measure was analysed separately using 3 x 2 robust ANOVA’s with 20\% trimmed means. These were again performed in R (R Core Team, 2016) using the t2way function from Wilcox (2016) in the WRS2 package (Mair et al., 2016). Posthoc tests were carried out using the mcp2atm function from the same package. 
For the People Details, there was a main effect of Group Type, $Q=20.86, p<.001$. Post-hoc tests revealed that the individuals and collaborative pairs recalled an equivalent number of these details ( $p=.09$ ) but both recalled less than the nominal pairs (both $p$ 's $<.001$ ). There was also a main effect of Recall Type, $Q=22.21, p<.001$, with participants recalling more People Details when using CCR than free recall. Finally, there was no Group Type x Recall Type interaction, $Q=0.23, p=.89$.

For the Action Details, there was a main effect of Group Type, $Q=25.76, p=.001$. Post-hoc tests revealed that the individuals and collaborative pairs recalled an equivalent number of these details $(p=.29)$ but both recalled less than the nominal pairs (both $p$ 's $<.001$ ). There was no main effect of Recall Type, $Q=0.03, p=.85$, and no Group Type $\mathrm{x}$ Recall Type interaction, $Q=1.48, p=.49$.

Finally, for the Environment Details, there was a main effect of Group Type, $Q=$ 26.91, $p<.001$. Post-hoc tests revealed that the individuals recalled fewer of these details than collaborative pairs ( $p=.004)$ and that both groups recalled fewer than the nominal pairs (both $p$ 's $<.006)$. There was also a main effect of Recall Type, $Q=23.11, p<.001$, with participants recalling more Environment Details when using CCR than free recall. Finally, there was no Group Type x Recall Type interaction, $Q=0.41, p=.82$.

To summarise the key findings, the collaborative groups recalled fewer People, Action, and Environment Details than the nominal groups, meaning CI occurred across all three categories of forensically relevant information. Whilst lone individuals recalled the least of each detail type, they were only significantly worse than the collaborative pairs when recollecting the Environment Details. Thus, the collaborative group advantage, at least in this exploratory analysis, is restricted in scope. Moreover, these findings demonstrated that the benefits of engaging in CCR over free recall are restricted to an enhanced recollection of People and Environment Details. 


\section{Recall Accuracy}

Few recall errors were made in this study ( $M=.72$ per condition), with many individuals, collaborative pairs, and nominal pairs not making any. The average number of recall errors (and association Standard Deviations) in each of the six conditions are in Table 1. The values are too small for any meaningful inferential analyses. Importantly, they are near identical across the six conditions, with the Mean number ranging from 0.35 to 1.15 , suggesting Group Type and Recall Type had no impact upon the number of errors made here.

\section{Discussion}

This study had three primary sets of findings. First, collaborative pairs of eyewitnesses took longer to recall a crime than lone eyewitnesses, but less time than nominal pairs of eyewitnesses. Second, the collaborative pairs of eyewitnesses recalled more correct information about a crime than lone eyewitnesses, but less than the nominal pairs of eyewitnesses. CI was therefore observed. Each of these effects occurred regardless of whether the participants recollected the crime using free recall or CCR. Combined, they show that having two co-witnesses collaboratively recall a crime can expedite the rate at which evidence is accumulated but doing so retards the volume of correct information remembered. Third, lone eyewitnesses, collaborative pairs, and nominal pairs all recalled more correct information about the crime when engaging in CCR than free recall. Importantly, few recall errors were made, irrespective of retrieval type. This third set of findings provides initial evidence to suggest that CCR may be a superior retrieval method that could be used as an alternative to free recall when interviewing eyewitnesses. Each set of findings is considered in more detail next.

\section{Time Taken to Recall the Crime}

It is important to know whether it is faster to take collaborative group statements about a crime (compared to individual statements) as situations can arise where large 
numbers of eyewitnesses need interviewing and evidence needs to be accumulated quickly to try and prevent a further offence being committed. It is unsurprising that collaborative and nominal pairs took longer to recall the crime than individuals, regardless of whether they used free recall or CCR, given that collaborative pairs typically remember more and nominal pairs require two separate retrieval attempts. It is also unsurprising that collaborative pairs took less time to recall the crime than two separate eyewitnesses, irrespective of the retrieval method used. This collaborative group advantage occurred as their members typically recalled a piece of information once whereas nominal group members, who worked independently, recalled some of the same information twice. In an exploratory analysis, it was also found that participants took longer to recall the crime using CCR than free recall, presumably because they recalled more when engaging in CCR. Combined, these findings show that asking multiple eyewitnesses to collaboratively recall a crime (at least in pairs) expedites the rate at which evidence is accumulated. Whilst this new finding is interesting, this knowledge may only be of practical relevance if collaboration has no negative impact upon the completeness and accuracy of the evidence recalled. That issue is discussed next.

\section{The Costs of Collaborative Memory}

The observation that the collaborative and nominal pairs recalled more correct information than lone individuals was expected and is consistent with past research showing groups generally recall more (Clark \& Stephenson, 1989; Wessel et al., 2015). A new finding here is that this effect occurred regardless of whether participants recollected the crime using free recall or CCR. In exploratory analyses, it was also found that the lone individuals and collaborative pairs correctly recalled an equivalent number of People and Action Details, but that the collaborative pairs correctly recalled significantly more Environment Details. This is the first evidence to suggest that collaboration may improve correct recall of less central/more peripheral crime details only and is an issue that warrants further investigation. 
The CI observed when collaborative pairs freely recalled the crime was expected as it is consistent with existing theory and research. To briefly recap, Basden et al.'s (1997) RSD theory suggests collaborative group members sometimes have idiosyncratic optimal retrieval strategies when freely recalling the same information, these different strategies disrupt each other, and this retards the group's recall. These effects are reduced (but not always eliminated) in smaller groups, as they have fewer conflicting retrieval strategies, and when group members are close acquaintances, as members who know each other well can sometimes use complementary retrieval practices (see Harris et al., 2011; Marion \& Thorley, 2016; Vredeveldt et al., 2016, 2017). CI is also reduced when there are study-test delays of more than 15 min (Abel \& Bäuml, 2017; Congleton \& Rajaram, 2011; Takahashi \& Saito, 2004). This has been attributed to a collaborative group advantage whereby all participants begin to forget the studied information but the collaborative group members, and not the nominal group members, can protect against this by cuing each other's memories of it (Abel \& Bäuml, 2017; Congleton \& Rajaram, 2011). Combined, these effects can explain why CI was present in earlier studies that had collaborative groups of previously unacquainted trios freely recall a crime after a 5 min study-test delay (Bärthel et al., 2017; Wessel et al., 2015) but it was absent in studies that had collaborative pairs, who were all or mostly close acquaintances, freely recall a crime after study-test delays of more than 15 min (Vredeveldt et al., 2016, 2017). Put simply, the latter two studies had designs that were optimal for eliminating CI. As the present study tested collaborative pairs of strangers after a $10 \mathrm{~min}$ study-test delay, two of the three conditions that promote CI were present during free recall, increasing the likelihood of CI occurring.

The CI observed when the collaborative pairs used CCR to recount the crime was not predicted. Instead, it was expected that CCR would align collaborative group members retrieval strategies and eliminate CI. That finding would have been consistent with those of 
earlier studies in the wider collaborative memory literature that aligned group members retrieval strategies and found no evidence of CI (Basden et al., 1997, Experiment 4; Finlay et al., 2000; Saraiva et al., 2016). There were methodological differences between the present study and these earlier studies that may explain why their (null) effects did not generalise here. In the present study, participants watched a crime video and recalled information from three broad categories, one category at a time. To recap, these categories related to the descriptions of the people involved in the crime, their actions, and the crime environment. Within these broad categories, there was potential for participants’ retrieval strategies to vary. For example, focussing on the first category, Witness A's preferred/optimal retrieval strategy may have involved describing the victim first whereas Witness B's may have involved describing the offender first. In Finlay et al. (2000) and Saraiva et al.'s (2016) studies, both group members were forced to recall all information in the exact same order, meaning their retrieval strategies were identical. In Basden et al.’s (1997, Experiment 4) study, which is more analogous to the present one as it also involved category-by-category recall, participants studied 6 categorised lists that each contained 15 words. Their categories contained far fewer pieces of information that those in the present study, perhaps decreasing the likelihood of participants in their study having conflicting retrieval strategies. Consistent with this suggestion, Basden et al. (1997, Experiment 1) found CI was less likely to occur when collaborative groups recalled categories with a small, compared to large, number of words. Thus, RSD may have been more prevalent in the present study's collaborative groups because of the large category sizes, causing CI. One way to test this possibility would be to further align collaborative group members retrieval strategies by having them recall from a smaller number of narrower retrieval categories. For example, it would be possible to divide the People Details into two separate categories: one relating to the victim and one relating to the perpetrator. Alternatively, Paulo et al.’s (2016, 2017) CCR categories could be adopted, 
as those authors utilised a larger number of narrower categories than the present study. If either approach reduced/eliminated CI, this would suggest the large category sizes in this study contributed towards the CI observed when collaborative groups recalled using CCR and demonstrate a practical approach for overcoming this problem (as narrower categories could be used in future).

Importantly, recall errors were rare in this study. Many individuals, collaborative pairs, and nominal pairs failed to make any, irrespective of retrieval type, and the average number was less than one per group. This is unsurprising as there was no attempt to induce recall errors in this study and free recall requires self-guided retrieval, meaning people typically only report information they are confident about (Koriat \& Goldsmith, 1994, 1996). Thus, whilst recall errors can occur in groups, and research shows that one group member's errors can contaminate other group members’ memory of a crime via memory conformity (see Wright et al., 2009), this study adds to the growing body of evidence suggesting that collaborative groups make few recall errors overall.

\section{Enhancing Eyewitness Memory with Category Clustering Recall}

A novel and important finding here is that individuals, collaborative pairs, and nominal pairs all remembered more correct information about the crime when recollecting using CCR, compared to free recall. This was tentatively predicted as previous studies had found recalling a crime using CCR, after previously recollecting it using free recall, can result in additional information, not generated during the initial free recall, being remembered (Paulo et al., 2016, 2017). The present study is, however, the first to examine CCR when it is the sole form of retrieval used and it is also the first to directly compare it to free recall. Exploratory analysis also showed that CCR enhanced the volume of People and Environment Details correctly recalled. Importantly, as mentioned, there were also few recall errors when using free recall or CCR. Combined, these findings provide initial evidence to suggest CCR 
is superior to free recall as a retrieval method. Future research directly comparing Paulo et al.’s (2016, 2017) original CCR to free recall would be beneficial as a similar CCR advantage would show the effect generalises even when there are variations to the procedure and it would further corroborate the technique’s superiority as a retrieval method.

It is beyond the scope of this study to determine why CCR was superior to free recall. Paulo et al. (2016, 2017) speculated that CCR is an effective retrieval strategy as people subjectively encode and store new information in semantic categories and asking them to recall information according to these categories may be less cognitively demanding for them, enhancing their recall. Moreover, Paulo et al. (2016, 2017) suggest that asking eyewitnesses to successively recall information from one category may induce spreading-activation that triggers memories of associated details that would have otherwise been forgotten. Further research is, however, required to establish whether these mechanisms can adequately explain why CCR was superior to free recall here.

\section{Practical Implications and Limitations}

There are two potentially important practical implications arising from this research. First, if the police needed to take statements from a group of unacquainted eyewitnesses in the immediate aftermath of a crime and were considering utilising collaborative recall to expedite evidence accumulation, then they would need to weigh up the costs and benefits of doing so. The current study shows that taking a statement from a collaborative pair is faster than taking two individual statements but that the collaborative group statement yields less information about the crime. In a practical sense, however, the extra time required to take two individual statements (based upon the nominal group average from the present study) was only 103.2 s. In everyday terms, that is not a substantial amount of time. Moreover, the volume of non-overlapping information correctly recalled about the crime increased by an average of 7 items (a 28.64\% increase). That extra information could be crucial in 
apprehending an offender. Thus, when there are two eyewitnesses, this speed-recall completeness trade-off seemingly favours taking individual statements over collaborative group statements. The speed-recall completeness trade-off may, however, favour taking collaborative group statements in criminal investigations with large numbers of eyewitnesses. For example, in the current study, taking 20 collaborative pairs’ statements took nearly 101 min, but taking 40 individual statements (using the nominal group data) took nearly 170 min. The former therefore offered more substantial time savings overall. These savings would increase further if there were more eyewitnesses. In real cases of this nature, the investigating officers would also compare/pool the statements taken, irrespective of whether individuals or collaborative pairs produced them. As the amount of information that can be recalled about an offence is not finite, and likely subject to ceiling effects, it is reasonable to speculate that a sufficiently large number of collaborative pairs (whose statements are pooled) could recall as much information about a crime as a comparable number of individuals (whose statements are pooled). It is beyond the scope of the current study to investigate this issue but future research examining the speed-recall completeness trade-off when statements are taken from different numbers of individuals and collaborative groups (e.g., two, ten, twenty, forty, etc), and when the information recalled by each group type is separately pooled, would be welcome as it could help inform police decision making regarding when it is most appropriate to take each type of statement.

The second practical implication is that it may be more profitable for the police to interview lone eyewitnesses using CCR instead of free recall, at least in the immediate aftermath of a crime, as doing so could increase the volume of correct information remembered without increasing the number of errors made. Indeed, if it was essential to have collaborative pairs of strangers recall a crime shortly after it occurred, it may be best to have them engage in CCR as this would produce more correct information than free recall. The 
downside to using CCR instead of free recall is that it would slow down the rate at which evidence is accumulated, although the current evidence suggests the extra time taken is not substantial (47.25 s for individuals, $67.5 \mathrm{~s}$ for collaborative pairs).

It is important to emphasise that this is the first study to examine the costs and benefits of collaborative eyewitness memory by having collaborative pairs of strangers, nominal pairs, and lone individuals watch a crime and recall it using free recall or CCR. It would be premature to make procedural recommendations based upon this limited evidence and further research confirming the reliability of these effects is welcome. It is also important not to overgeneralise these findings to more formal police interviews. In practice, the experience of recalling a crime during a formal police interview is very different to the experience of recalling it in this study. Unlike the interviews in the present study, formal police interviews typically involve one individual eyewitnesses only, they usually take place after a delay (as the eyewitnesses need to attend a police station), they can have several distinct stages (e.g., free recall followed by witness compatible questioning), and can incorporate a range of different mnemonics that aid recall (e.g., context reinstatement). Indeed, free recall and CCR have never been directly compared when they form part of a formal police interview, such as the Cognitive Interview (as in Paulo et al., 2016, 2017), so their relative effectiveness in these contexts, irrespective of whether individuals or collaborative groups are interviewed, is unknown. Further research examining how well individuals and collaborative groups of eyewitnesses can recall crimes during formal police interviews, when free recall or CCR is used, is therefore essential before generalisations to these contexts are made.

There are also several aspects of the current study that restrict its external validity. The sample of participants used was quite homogenous (individuals who responded to University advertisements) and memory of a single crime video was assessed. A re- 
examination of the effects observed here with a less homogenous sample and different crime video would be welcome to test their generalisability. The study also focussed on collaborative pairs of strangers only, with all participants experiencing a 10-min study-test delay. As discussed, CI is sensitive to changes in collaborative group size, the social relationship of group members, and study-test delays. It is possible that a different pattern of results would have been observed if any of these factors had differed. For example, CI may not have been observed in this study if there was a longer study-test delay. Further research examining how each of these variables impacts upon collaborative eyewitness memory, and memory performance when CCR is used, would also be welcome.

Finally, it is important to acknowledge that this study did not examine the impact of group-level collaborative retrieval strategies on CI. For example, some collaborative pairs may have opted to recall the crime in a turn-taking fashion (i.e., one member recalls one piece of information at a time, regardless of whether freely recalling the crime or recalling from categories), whereas others may have opted to recall items in a free-flowing fashion. Previous research suggests the former group-level retrieval strategy promotes CI (Marion \& Thorley, 2016). Other types of group-level strategies may also be important. Similarly, the impact of collaborative group members' communication styles on CI was not examined. This issue has previously been studied in relation to pilots' recall of flight scenarios (Meade, Nokes, \& Morrow, 2009), elderly couples’ recall of autobiographical events (Harris et al., 2011), middle-aged couples' recall of a crime during a theatre play (Vredeveldt et al., 2016), and students' recall of a violent videotaped incident (Vredeveldt et al., 2017). Whilst these studies have produced a small number of conflicting findings, they have generally found that collaborative pairs who actively repeat, rephrase, and elaborate upon each other's statements recall more information than those who do not and this can occasionally result in collaborative groups recalling more than nominal groups. There were a small number of 
instances in the current study where collaborative groups recalled more than the nominal groups so a closer examination of such cases in future, to determine whether the collaborative groups employed common group-level retrieval strategies or had common communication styles, would be welcome as it may offer an insight into how collaborative recall can be maximised (and CI potentially eliminated) in everyday contexts. For example, if CI was lowest in any collaborative groups whose members took turns to recall information, future collaborative groups of eyewitnesses could be encouraged to recall in this manner.

\section{Conclusion}

This study provides important new insights into the costs and benefits of collaborative eyewitness memory and also provides initial evidence for a promising new retrieval strategy that could enhance eyewitnesses' recall of crimes. Focussing on the former, the study identified a speed-recall completeness trade-off when asking pairs of previously unacquainted eyewitnesses to collaboratively recall a crime in its immediate aftermath. Whilst taking individual statements from both group members takes a little longer, a larger body of evidence is obtained. Thus, the speed-recall completeness trade-off likely favours taking separate statements when there are only two eyewitnesses (but, as discussed, it potentially favours taking collaborative group statements when there are larger numbers of eyewitnesses). Focussing on the latter, this study identified CCR as being a potentially superior retrieval strategy to free recall, in the sense that it produces a more complete account of crimes, and further research on CCR's benefits is encouraged. If CCR proves to be superior, it could offer police investigators a simple, but effective, way of enhancing eyewitnesses’ memory of crimes. 


\section{References}

Abel, M. \& Bäuml, K.-H. T. (2017). Collaborative remembering revisited: Study context access modulates collaborative inhibition and later benefits for individual memory. Memory \& Cognition, 45(8), 1319-1334. doi:10.3758/s13421-017-0737-9

Andersson, J., \& Rönnberg, J. (1995). Recall suffers from collaboration: Joint recall effects of friendship and task complexity. Applied Cognitive Psychology, 9(3), 199-211. doi: 10.1002/acp.2350090303

Andersson, J., \& Rönnberg, J. (1996). Collaboration and memory: Effects of dyadic retrieval on different memory tasks. Applied Cognitive Psychology, 10(2), 171-181. doi: 10.1002/(SICI)1099-0720(199604)10:2

Barber, S. J., Harris, C. B., \& Rajaram, S. (2015). Why two heads apart are better than two heads together: Multiple mechanisms underlie the collaborative inhibition effect in memory. Journal of Experimental Psychology: Learning, Memory, and Cognition, 41(2), 559-566. doi:10.1037/xlm0000037

Bärthel, G. A., Wessel, I., Huntjens, R. J., \& Verwoerd, J. (2017). Collaboration enhances later individual memory for emotional material. Memory, 25(5), 636-646. doi: $0.1080 / 09658211.2016 .1208248$

Basden, B. H., Basden, D. R., Bryner, S., \& Thomas, R. L. (1997). A comparison of group and individual remembering: Does collaboration disrupt retrieval strategies? Journal of Experimental Psychology: Learning, Memory, and Cognition, 23(5), 1176-1176-1189. doi:10.1037/0278-7393.23.5.1176

Basden, B. H., Basden, D. R., \& Henry, S. (2000). Costs and benefits of collaborative remembering. Applied Cognitive Psychology, 14(6), 497-497-507. doi:10.1002/10990720(200011/12)14:6<497::AID-ACP665>3.0.CO;2-4

Clark, N. K., \& Stephenson, G. M. (1989). Group remembering. In P. B. Paulus 
(Ed.), Psychology of Group Influence (pp. 357-391). Hillsdale, NJ: Lawrence Erlbaum Associates.

Collins, A. M., \& Loftus, E. F. (1975). A spreading-activation theory of semantic processing. Psychological Review, 82(6), 407-428. doi:10.1016/B978-1-4832-1446-7.50015-7

Congleton, A. R., \& Rajaram, S. (2011). The influence of learning methods on collaboration: Prior repeated retrieval enhances retrieval organization, abolishes collaborative inhibition, and promotes post-collaborative memory. Journal of Experimental Psychology: General, 140(4), 535-535-551. doi:10.1037/a0024308

Dalrymple-Alford, E. C., \& Aamiry, A. (1969). Language and category clustering in bilingual free recall. Journal of Verbal Learning and Verbal Behavior, 8(6), 762-768. doi:10.1016/S0022-5371(69)80041-1

Finlay, F., Hitch, G. J., \& Meudell, P. R. (2000). Mutual inhibition in collaborative recall: Evidence for a retrieval-based account. Journal of Experimental Psychology: Learning, Memory, and Cognition, 26(6), 1556-1556-1567. doi:10.1037/0278-7393.26.6.1556

Fisher, R. P., \& Geiselman, R. E. (1992). Memory-Enhancing Techniques for Investigative Interviewing: The Cognitive Interview. Springfield, IL: Charles C. Thomas.

Gabbert, F., Memon, A., \& Allan, K. (2003). Memory conformity: Can eyewitnesses influence each other's memories for an event? Applied Cognitive Psychology, 17(5), 533-543. doi:10.1002/acp.885

Gabbert, F., Wright, D. B., Memon, A., Skagerberg, E. M., \& Jamieson, K. (2012). Memory conformity between eyewitnesses. Court Review, 48, 36-138.

Geiselman, R. E., \& Fisher, R. P. (2014). Interviewing witnesses and victims. In M. St-Yves (Ed.), Investigative Interviewing: Handbook of Best Practices (pp. 29-63). Toronto, ON: Thomson Reuters. 
Harris, C. B., Barnier, A. J., \& Sutton, J. (2012). Consensus collaboration enhances group and individual recall accuracy. The Quarterly Journal of Experimental Psychology, 65(1), 179-194. doi:10.1080/17470218.2011.608590

Harris, C. B., Barnier, A. J., \& Sutton, J. (2013). Shared encoding and the costs and benefits of collaborative recall. Journal of Experimental Psychology: Learning, Memory, and Cognition, 39(1), 183-195. doi:10.1037/a0028906

Harris, C. B., Keil, P. G., Sutton, J., Barnier, A. J., \& McIlwain, D. J. F. (2011). We remember, we forget: Collaborative remembering in older couples. Discourse Processes, 48(4), 267-303. doi:10.1080/0163853X.2010.541854

Houston, K. A., Clifford, B. R., Phillips, L. H. \& Memon, A. (2013). The emotional eyewitness: The effects of emotion on specific aspects of eyewitness recall and recognition performance. Emotion, 13(1), 118-128. doi:10.1037/a0029220

Hyman, I. E., Jr., Cardwell, B. A., \& Roy, R. A. (2013). Multiple causes of collaborative inhibition in memory for categorised word lists. Memory, 21(7), 875-890. doi:10.1080/09658211.2013.769058

Koriat, A., \& Goldsmith, M. (1994). Memory in naturalistic and laboratory contexts: distinguishing the accuracy-oriented and quantity-oriented approaches to memory assessment. Journal of Experimental Psychology: General, 123(3), 297-315. doi:10.1037/0096-3445.123.3.297

Koriat, A., \& Goldsmith, M. (1996). Monitoring and control processes in the strategic regulation of memory accuracy. Psychological Review, 103(3), 490-517. doi: 10.1037/0033-295X.103.3.490

Mair, P., Schoenbrodt, F., \& Wilcox, R. (2016). WRS2: Wilcox robust estimation and testing.

Manning, J. R., \& Kahana, M. J. (2012). Interpreting semantic clustering effects in free 
recall. Memory, 20(5), 511-517. doi:10.1080/09658211.2012.683010

Marion, S. B., \& Thorley, C. (2016). A meta-analytic review of collaborative inhibition and postcollaborative memory: Testing the predictions of the retrieval strategy disruption hypothesis. Psychological Bulletin, 142(11), 1141-1164. doi:10.1037/bul0000071

Meade, M. L., Nokes, T. J., \& Morrow, D. G. (2009). Expertise promotes facilitation on a Collaborative memory task. Memory, 17(1), 39-48. doi:10.1080/09658210802524240

National Institute of Justice (1999). Eyewitness evidence: A guide for law enforcement. Retrieved from https://www.ncjrs.gov/pdffiles1/nij/178240.pdf

Paterson, H. M., \& Kemp, R. I. (2006). Co-witnesses talk: A survey of eyewitness discussion. Psychology, Crime, \& Law, 12(2), 181-191. doi:10.1375/pplt.12.2.424

Paulo, R. M., Albuquerque, P. B., \& Bull, R. (2015). The enhanced cognitive interview: Expressions of uncertainty, motivation and its relation with report accuracy. Psychology, Crime \& Law, 22(4), 366-381. doi:10.1080/1068316X.2015.1109089

Paulo, R. M., Albuquerque, P. B., \& Bull, R. (2016). Improving the enhanced cognitive interview with a new interview strategy: Category clustering recall. Applied Cognitive Psychology, 30(5), 775-784. doi:10.1002/acp.32532016

Paulo, R. M., Albuquerque, P. B., Vitorino, F., \& Bull, R. (2017). Enhancing the cognitive interview with an alternative procedure to witness-compatible questioning: category clustering recall. Psychology, Crime \& Law. doi:10.1080/1068316X.2017.1351966

R Core Team (2016). R: A language and environment for statistical computing. $R$ Foundation for Statistical Computing, Vienna, Austria. Retrieved from https://www.rproject.org/.

Rajaram, S. (2011). Collaboration both hurts and helps memory: A cognitive perspective. Current Directions in Psychological Science, 20(2), 76-81. doi:10.1177/0963721411403251 
Rajaram, S., \& Pereira-Pasarin, L. P. (2010). Collaborative memory: Cognitive research and theory. Perspectives on Psychological Science, 5(6), 649-663. doi:10.1177/1745691610388763

Robinson, J. A. (1966). Category clustering in free recall. The Journal of Psychology Interdisciplinary and Applied, 62(2), 279-286. doi:10.1080/00223980.1966.10543793

Roediger, H. L., Meade, M. L., \& Bergman, E. T. (2001). Social contagion of memory. Psychonomic Bulletin \& Review, 8(2), 365-371. doi:10.3758/BF03196174

Ross, M., Spencer, S. J., Blatz, C. W., \& Restorick, E. (2008). Collaboration reduces the frequency of false memories in older and younger adults. Psychology and Aging, 23(1), 85-92. doi:10.1037/0882-7974.23.1.85

Saraiva, M., Albuquerque, P. B., \& Arantes, J. (2016). Elimination of collaborative inhibition effect using the Method of Loci. Psicothema, 28(2), 181-186 doi:10.7334/psicothema2015.241

Skagerberg, E. M., \& Wright, D. B. (2008). The prevalence of co-witnesses and co-witness discussions in real eyewitnesses. Psychology, Crime, \& Law, 14(6), 513-521. doi:10.1080/10683160801948980

Takahashi, M., \& Saito, S. (2004). Does test delay eliminate collaborative inhibition? Memory, 12(6), 722-731. doi:10.1080/09658210344000521

Thorley, C., \& Dewhurst, S.A. (2007). Collaborative false recall in the DRM procedure: Effects of group size and group pressure. European Journal of Cognitive Psychology, 19(6), 867-881. doi:10.1080/09541440600872068

Thorley, C., \& Dewhurst, S. A. (2009). False and veridical collaborative recognition. Memory, 17(1), 17-25. doi:10.1080/09658210802484817

Vredeveldt, A., Groen, R. N., Ampt, J. E., \& Van Koppen, P. J. (2017). When discussion 
between eyewitnesses helps memory. Legal and Criminological Psychology, 22(2), 242-549. doi:10.1111/lcrp.12097

Vredeveldt, A., Hildebrandt, A., \& Van Koppen, P. J. (2016). Acknowledge, repeat, rephrase, elaborate: Witnesses can help each other remember more. Memory, 24(5), 669-682. doi:10.1080/09658211.2015.1042884

Weigold, A., Russell, E. J., \& Natera, S. N. (2014). Correction of false memory for associated word lists by collaborating groups. The American Journal of Psychology, 127(2), 183190. doi:10.5406/amerjpsyc.127.2.0183

Weldon, M. S., \& Bellinger, K. D. (1997). Collective memory: Collaborative and individual processes in remembering. Journal of Experimental Psychology: Learning, Memory, and Cognition, 23(5), 1160-1175. doi:10.1037/0278-7393.23.5.1160

Wessel, I., Zandstra, A. R. E., Hengeveld, H. M. E., \& Moulds, M. L. (2015). Collaborative recall of details of an emotional film. Memory, 23(3), 437-444.

doi:10.1080/09658211.2014.895384

Wilcox, R. R. (2016). Understanding and applying basic statistical methods using $R$. Hoboken, NJ: John Wiley \& Sons.

Wright, D.B., Memon, A., Skagerberg, E.M., \& Gabbert, F. (2009). When eyewitnesses talk. Current Directions in Psychological Science, 18(3), 174-178. doi: 10.1111/j.14678721.2009.01631.x

Wright, D. B., Self, G., \& Justice, C. (2000). Memory conformity: Exploring misinformation effects when presented by another person. British Journal of Psychology, 91(2), 189202. doi:10.1348/000712600161781 
Table 1

Means (and associated Standard Deviations) showing the overall number of details correctly and incorrectly recalled about a crime by individual participants, collaborative pairs, and nominal pairs who engaged in either free recall or category clustering recall. Also shown are the number of details correctly recalled about the people involved in the crime, their actions, and the crime environment. These latter three values combine to form the overall number of details correctly recalled

\begin{tabular}{|c|c|c|c|c|c|c|c|c|c|c|c|}
\hline \multirow[t]{2}{*}{ Group type } & \multirow[t]{2}{*}{ Recall Type } & \multicolumn{2}{|c|}{ Overall Correct } & \multicolumn{2}{|c|}{ People Details } & \multicolumn{2}{|c|}{ Action Details } & \multicolumn{2}{|c|}{ Environment Details } & \multicolumn{2}{|c|}{ Overall Errors } \\
\hline & & $\underline{M}$ & $\underline{S D}$ & $\underline{M}$ & $\underline{S D}$ & $\underline{M}$ & $\underline{S D}$ & $\underline{M}$ & $\underline{S D}$ & $\underline{M}$ & $\underline{S D}$ \\
\hline \multirow[t]{2}{*}{ Individual } & Free Recall & 18.25 & 7.48 & 6.35 & 2.72 & 9.30 & 4.22 & 2.60 & 2.26 & 0.50 & 0.76 \\
\hline & Category Clustering & 22.40 & 6.45 & 9.00 & 3.23 & 9.30 & 3.19 & 4.10 & 2.57 & 0.65 & 0.87 \\
\hline \multirow[t]{2}{*}{ Collaborative } & Free Recall & 22.85 & 9.24 & 8.80 & 4.77 & 10.75 & 4.93 & 3.30 & 2.10 & 0.35 & 0.74 \\
\hline & Category Clustering & 26.55 & 6.04 & 10.40 & 3.23 & 10.60 & 3.68 & 5.55 & 2.19 & 0.80 & 1.15 \\
\hline \multirow[t]{2}{*}{ Nominal } & Free Recall & 28.70 & 9.47 & 9.90 & 2.88 & 13.40 & 4.79 & 5.40 & 3.65 & 0.95 & 1.15 \\
\hline & Category Clustering & 34.85 & 7.40 & 12.10 & 3.90 & 15.75 & 4.34 & 7.00 & 2.58 & 1.15 & 1.09 \\
\hline
\end{tabular}

Note: The Means presented here are untrimmed 